\title{
Salud mental y resocialización: tensiones y desafíos
}

Omar Alejandro Bravo

\section{Resumen}

Las prisiones latinoamericanas se caracterizan por altos niveles de hacinamiento y violencia institucional, así como por contener a una mayoría de jóvenes oriundos de contextos de pobreza y vulnerabilidad. De la misma forma, el encarcelamiento afecta a la salud mental de esas personas, en función de la violencia cotidiana, la falta de privacidad, la distancia con la familia y el ocio forzado, siendo el uso de drogas una forma de amenizar ese sufrimiento.

No obstante, los efectos del encarcelamiento en la salud mental no han sido suficientemente abordados, en parte porque el papel de la psicología en esos contextos ha sido sofocado por la demanda de contribuir para los procesos de resocialización de la población carcelaria. Esta resocialización, cuando no se ofrecen condiciones de estudio, capacitación laboral y posibilidades de inserción social futura, se torna un imperativo vacío, una ficción necesaria a la continuidad de este tipo de instituciones.

Este texto, a manera de ensayo, intenta mostrar las tensiones y contradicciones entre los objetivos de resocialización que la prisión impone y una perspectiva de salud mental que dimensione formas de relación solidarias y amplias, tomando como referencia para eso una experiencia de intervención realizada en la prisión de Villahermosa, en Cali, Colombia.

Palabras llave: salud mental; prisiones; resocialización.

\section{Saúde mental e ressocialização: tensões e desafíos}

\section{Resumo}

As prisões latino-americanas caracterizam-se por altos níveis de superlotação e violência institucional, bem como por abrigar uma maioria de jovens oriundos de contextos de pobreza e vulnerabilidade. Da mesma forma, o encarceramento afeta a saúde mental dessas pessoas, em decorrência da violência cotidiana, da falta de privacidade, do afastamento da família e do ócio forçado, sendo o uso de drogas uma forma de amenizar esse sofrimento.

No entanto, os efeitos do encarceramento na saúde mental não têm sido suficientemente abordados, em parte porque o papel da psicologia nesses contextos tem sido sufocado pela demanda de contribuir para os processos de ressocialização da população prisional. Esta ressocialização, quando não se oferecem condições de estudo, formação laboral e posibilidades de inserção social futura, torna-se um imperativo vazio, uma ficção necessária à continuidade deste tipo de instituições.

Este texto, a maneira de ensaio, tenta mostrar as tensões e contradições entre os objetivos de ressocialização que a prisão impõe e uma perspectiva de saúde mental que dimensione formas de relação solidárias e amplas, tomando como referência para isso uma experiência de intervenção realizada na prisão de Villahermosa em Cali, Colômbia.

Palavras - chave: saúde mental; prisões; ressocialização

1 Director Maestría en Intervención Psicosocial, Universidad Icesi, Cali, Colombia. Correo: oabravo@icesi.edu.co 
Mental health and resocialization: tensions and challenges

\section{Abstract}

Latin American prisons are characterized by overcrowding and institutional violence. Also, inmates are usually young adults who used to live in contexts of poverty and vulnerability. Incarceration affects their mental health because of daily violence, lack of privacy, distance from family and forced leisure, and drug abuse has become a way to alleviate this suffering, However, incarceration effects on mental health have not been sufficiently addressed because psychology has been primarily focused on inmate's resocializing. Considering that study options, job training, and possibilities of future social insertion are not offered, resocializing becomes an empty task and a necessary fiction for the continuity of this type of institution.

This text, as an essay, points out the tensions and contradictions between resocializing objectives that the prison imposes and offers a mental health perspective that takes in account forms of solidarity and broad relationships. The essay is based on an experience of intervention carried out in the Villahermosa prison in Cali, Colombia.

Keywords: mental health; prisons; resocialization

\section{Introducción}

Cualquier referencia a los sistemas penitenciarios latinoamericanos suele acompañarse de una mención a sus numerosos problemas, con frecuencia sintetizados en el término crisis.

Esa caracterización engloba entonces a varios aspectos del funcionamiento de estas instituciones, entre ellos, el hacinamiento presente en la mayoría de las cárceles, las malas condiciones de acceso a la salud, educación y capacitación laboral, los elevados índices de violencia institucional y entre la propia población reclusa y los importantes márgenes de reincidencia, principalmente.

El hacinamiento en particular implica, según Matthews (2011), que

Los internos permanezcan más tiempo en sus celdas; disminuya el acceso al trabajo y formación en las prisiones; se incrementen los problemas de dis- ciplina y seguridad; aumente la tensión o violencia entre los internos; se afecten las relaciones entre los internos y los funcionarios de prisiones. (p. 302).

En este contexto, los requerimientos mínimos para un posible funcionamiento de las cárceles, señalados por Carranza (2018), (espacio físico y suficiente personal penitenciario) están lejos de cumplirse. Todos estos problemas señalados están atravesados por otros, de carácter estructural y que excede en parte al propio sistema carcelario: la gran mayoría de los presos/as latinoamericanos proviene de contextos de exclusión y pobreza, tienen baja escolaridad, son jóvenes y afrodescendientes (CARRANZA, 2018).

Un informe del Banco Latinoamericano del Desarrollo (BID, 2020), describe esta situación general de forma apropiada:

América Latina y el Caribe (ALC) tiene un millón $\mathrm{y}$ medio de reclusos y una tasa de encarcelamiento que se ha duplicado desde el año 2000: ha aumentado un $120 \%$ frente a un tímido $24 \%$ en el resto del mundo. Estos datos son fruto de un sistema de justicia que, en líneas generales, opta por el encarcelamiento como principal medida punitiva en lugar de explorar otras opciones como la libertad vigilada, los servicios comunitarios o las penas económicas. (p. 4).

Las ventajas de privilegiar las penas alternativas al encarcelamiento han sido ampliamente demostradas; no obstante, el afán punitivista de los gobiernos y sistemas penales latinoamericanos sigue priorizando el encierro, la medida más infectiva y cara (BRAVO \& CASTRILLÓN, 2010).

Estos problemas mencionados atraviesan la propia historia de las cárceles, pudiendo ubicarse su origen en el período colonial (GRILLO, 1980; AGUIRRE, 2009), y ha resistido a las numerosas propuestas de cambio y recomendaciones hechas por organismos internacionales y especialistas, siendo el documento de Naciones Unidas, titulado Reglas Mínimas de las Naciones Unidas para el Tratamiento de los Reclusos, Reglas Nelson Mandela, una síntesis apropiada de estos reclamos y propuestas (ONU, 2015).

La pandemia del Covid 19 vino a agravar este preocupante escenario (ILANUD, 2020), dado que las personas privadas de libertad, por la propia 
situación de hacinamiento, se ven particularmente expuestas a cualquier enfermedad transmisible por contacto interpersonal. Así mismo, las restricciones a las visitas, dispuestas de forma preventiva, aumentaron los problemas relacionados a la distancia y el aislamiento social, con previsibles repercusiones en la salud mental de los internos.

En lo que hace a la salud mental en particular, las consecuencias de sufrir esas condiciones de maltrato señaladas, producen formas de sufrimiento psíquico que se expresan de manera diferenciada en cada sujeto, pero que responden en general a la falta de intimidad y espacios personales, el ocio forzado, la violencia cotidiana y la incertidumbre acerca del futuro una vez cumplida la pena (CALVO, 2014; NIÑO, DÍAZ \& RAMÍREZ, 2017; BRAVO, 2018).

El sistema penitenciario colombiano expresa, de forma particular, esta realidad general descripta. En este país, las personas privadas de libertad ascienden a 97.624, siendo 90.631 hombres y 6.993 mujeres, con un porcentaje de hacinamiento de $20,7 \%$. De ese total, 20.648 sujetos están en condición de sindicados/as, a la espera de juicio (INPEC, 2021).

La composición parcialmente diferenciada de la población carcelaria colombiana tiene que ver con la persistencia del conflicto armado y del narcotráfico y sus efectos en las condenas relacionadas a esos delitos. Así mismo, y parcialmente vinculada a esta singularidad señalada, las formas de organización interna de los presos de los pabellones de las distintas prisiones se basan en la conformación de grupos violentos que dominan al resto de la población reclusa y les imponen sus reglas, que incluyen el pago por el acceso a un lugar relativamente cómodo para dormir, por ejemplo. Estas prácticas multiplican las consecuencias perjudiciales del encierro y conforman una especie de doble poder (el de la institución y el de los propios detenidos), lo que aumenta las condiciones de opresión y maltrato (BRAVO, 2012). Por otra parte, el acceso a educación y capacitación laboral es muy deficiente, así como los servicios de salud.

El propósito de este texto es considerar, a manera de ensayo y a partir de la experiencia de investigación e intervención del autor en una cárcel de la ciudad de Cali, Colombia, la necesidad de pensar una crítica a las instituciones carcelarias y sus efectos, desde la perspectiva de la salud mental comunitaria. Esta reflexión, a pesar de formulada en otros textos de propia autoría (BRAVO, 2012; 2016; 2018; BRAVO \& CASTRILLÓN, 2010) y de haber sido realizada también a partir de diversas investigaciones en este campo (CALVO, 2014; LOMBRAÑA, 2016; BEDRIÑANA, et al, 2017; NIÑO, DÍAZ Y RAMÍREZ, 2017), parte de suponer la insuficiencia de la crítica realizada hasta aquí a esas instituciones y sus consecuencias. Esto implica necesariamente referir a la resocialización, por ser este el objetivo formal y declarado de las cárceles, que se mantiene a pesar de los elevados índices de reincidencia y las condiciones de encierro ya señaladas, o sea, de su propio fracaso.

\section{Resocialización y salud mental: caminos diferen- ciados}

Las instituciones surgidas de la modernidad se propusieron brindar elementos para una inclusión social plena y efectiva, siendo la educación y el trabajo dos de los principales motores de esa empresa, dentro de una expectativa de progreso social, en general supuesta como lineal y sin conflictos. De esta manera confluyeron diversos tipos de instituciones, surgidas todas de este período y propósitos, como las escuelas, los hospitales psiquiátricos o las propias cárceles.

En las cárceles en particular la resocialización es el objetivo principal y la propia razón de su existencia, al menos en términos formales, y que se inscribe en esa expectativa institucional general mencionada.

De la Cuesta Arzamendi (1993) menciona dos modelos principales de resocialización: el relacionado al enfoque funcionalista, donde se procura "corregir o rellenar esa carencia o defectos de socialización" (p. 11) y el enfoque valorativo, que considera a la conducta delictiva "una manifestación de la incapacidad del delincuente de autodeterminarse y de controlar su comportamiento" (p. 12).

Ambas posturas son claramente insuficientes para explicar el fenómeno del que trata, mucho más para guiar un posible tratamiento penal. En general, los criterios utilizados se ubican en una posición intermedia entre ambos paradigmas, lo que no reduce las críticas frente a lo que se considera una ideología terapéutica basada en una ficción.

Jiménez (2017) direcciona estas críticas al sistema penitenciario colombiano, a partir de consi- 
derar a ese propósito resocializador una frustración, apoyada también en la paradoja de educar para la libertad a partir de la privación de la misma.

De alguna manera, estos principios de la resocialización mantienen puntos de contacto con el tratamiento moral pineliano, primera tentativa de diferenciar y tratar a la locura en el inicio de la revolución francesa. La proximidad tiene que ver con una suposición común: hay un déficit moral que produce un disfuncionamiento social y personal. Este déficit sería corregible a través de incentivos morales, basados en una mezcla de puniciones y premios, que devolverían al sujeto las condiciones para su inserción social de forma productiva.

Ambas expectativas y formas de intervención parten también de un reduccionismo particular: existiría una única identidad de loco/a, así como una de delincuente, no formalizada quizás en una definición explícita pero deducible a partir del tratamiento común otorgado. Esta ficción se sostiene con mucha dificultad, dada la diversidad de formas de sufrimiento psíquico y sujetos que se mezclan en una institución manicomial, así como lo forzado que resulta pensar en una única definición de delincuente (más allá de una caracterización legal), ya que el sentido del acto y las cuestiones subjetivas implicadas en un asesinato, un robo, un delito sexual o una estafa son muy diferentes, así como difieren inclusive dentro de cada tipo penal en lo que hace a motivaciones y circunstancias. Proponer entonces un tratamiento institucional único para esta diversidad de acciones y sujetos, tropieza necesariamente con una serie de dificultades previsibles.

En este orden, y asociado a ese objetivo de la resocialización, el denominado tratamiento penitenciario se define como "un conjunto de actividades asistenciales y terapéuticas, que deben ofrecerse al interno, al interior de la cárcel, y que están dirigidas a conseguir los fines previstos por la ley de ejecución penal” (CESANO, 2007, p. 66). Más allá de mantener una perspectiva interdisciplinaria y proponer un tratamiento individualizado, las propias condiciones de las cárceles, las colombianas en este caso, tornan este propósito imposible.

Por todo esto, y pesar del tiempo transcurrido desde su publicación, el trabajo de Goffman (2001) sigue siendo, en este sentido, una referencia inevitable. La definición de institución total, expresada en ese texto, es entendida como "lugar de residencia y trabajo, donde un gran número de individuos en igual situación, aislados de la sociedad por un periodo apreciable de tiempo, comparten en su encierro una rutina diaria administrada formalmente" (p. 13).

De esta forma, se engloban manicomios, cárceles y conventos, entre otros, parcialmente diferenciables en función de la población que contienen y las diferentes expectativas institucionales asociadas (curar, resocializar, cuidar, etc.), pero equiparables en cuanto a funcionamiento y efectos posibles.

En lo que hace al funcionamiento, los puntos en común tienen que ver con la existencia de actividades cotidianas impuestas y homogéneas, la falta de privacidad, la distancia con la familia y el mundo externo en general y la manera en que se organizan el tiempo y el espacio, lo que para Goffman lleva a la conformación de un mundo propio del interno.

Este análisis goffmaniano permite introducir la cuestión de la salud mental, en parcial contrapunto con esas expectativas institucionales y funcionamiento de las instituciones totales y, en particular, la de la resocialización.

La salud mental, según la Organización Mundial de la Salud, debería referir a un completo estado de bienestar, tanto físico como mental. Esta definición ha sido posteriormente objeto de críticas, por referir a una meta ideal, imposible de alcanzar y sostener (LOPERA-ECHAVARRÍA, 2014).

Desde dichas críticas, otro tipo de conceptualizaciones de este campo se han ido produciendo. En este orden, Galende (1998), considera a la salud mental como

un ámbito multidisciplinario destinado a prevenir, asistir y propender a la rehabilitación de los padecimientos mentales, y lo hace desde una comprensión de los lazos sociales deseables, implementando determinadas políticas dirigidas a la integración social y comunitaria de los individuos involucrados. (GALENDE, 1998, p. 108).

Cabe destacar en esta definición la mención a los padecimientos mentales, en lugar de trastornos mentales o enfermedad mental. De esta forma, se toma distancia del modelo médico dicotómico, que traza una frontera estricta entre la salud y la enfermedad, entendiendo de esta manera que el padecimiento mental (también posible de entender como 
sufrimiento psíquico) refiere a formas posibles de transitar la existencia, eventualmente sujetas a esas manifestaciones en función de acontecimientos de la vida, que impactan en cada sujeto de manera diferenciada.

Relacionar el sufrimiento psíquico con las condiciones de vida de una persona, lleva a referenciar aspectos sociales, económicos y culturales, que tanto pueden favorecer esas manifestaciones como ofrecer condiciones para su contención y superación. Desde esta perspectiva se entiende el uso del adjetivo comunitaria que suele acompañar el término salud mental, al menos cuando se lo considera desde este enfoque ampliado.

La relativa hegemonía alcanzada por este tipo de políticas que ha llevado, por ejemplo, al cierre de numerosas instituciones manicomiales, se ve confrontada con el aumento de la administración de la medicación psiquiátrica, en tipos de drogas y cantidad. Los manuales de referencia diagnóstica del tipo DSM operan como respaldo práctico para estas políticas al patologizar aspectos de la vida cotidiana, desde un criterio psicopatológico que intenta presentarse como ateórico (DESVIAT, 2016). De alguna forma, se trata de una política manicomial sin muros, donde la violencia del encierro es suplantada por la alienación derivada del exceso en la medicalización.

Esta tensión entre ambos modelos refiere fundamentalmente a una discusión que es, en definitiva, política, ya que refiere a formas de organización social que diferencian y privilegian el control, en un caso, y el cuidado, en el otro.

Desde estos señalamientos, es posible proyectar ese paradigma de la salud mental comunitaria hacia la tensa relación que supone con el modelo de resocialización que guía a las políticas y el tratamiento penitenciario.

Esta relación, a partir de la definición de salud mental ya citada y la referencia anterior a Goffman, transita por un camino predecible, que podría resumirse en la crítica a los efectos del encierro y sus efectos en la salud mental de las personas que lo sufren y las consecuencias del mismo, una vez cumplida la pena. En este sentido podría afirmarse, inclusive, que el encarcelamiento, así como el encierro manicomial, tiende a promover un nuevo tipo de socialización signada por una identidad asociada al estigma (loco o delincuente) y que favorece formas de relación asociadas a ese trazo único que, en el caso de la población carcelaria, puede incidir en la repetición de conductas ilegales.

Por esto, cabe reiterar (BRAVO 2012; 2016) la desproporción política en relación a las críticas hechas a las instituciones manicomiales con relación a las que se dirigen a las cárceles, al menos desde el campo de la salud mental. De manera especulativa, podría suponerse que esta disparidad tendría que ver con la falta de alternativas claras que promuevan otras formas de tramitar las infracciones a la ley, más allá de propuestas insuficientes e incompletas hechas desde el área del derecho, fundamentalmente. Dichas alternativas, en lo que hace a las políticas manicomiales, existen desde hace tiempo y tienen una fuerte expresión institucional, como ya fue señalado. Se trataría, en definitiva y siguiendo la línea trazada por Basaglia (1972) de negar la institución, lo que significa abandonar la ilusión de su posible mejoramiento, para sostener la necesidad de un cambio radical en su estructura y sentido.

Por todo esto, y de acuerdo con Galende (1990) resultaría más apropiado hablar de integración que de resocialización, ya que la primera refiere a una acción en doble sentido donde el individuo que tuvo sus lazos sociales afectados pueda integrarse a la comunidad y al mismo tiempo, la comunidad se disponga a integrarlo. Esta posibilidad es inseparable de las condiciones sociales y económicas que deben permitir ese proceso y toma distancia de la noción anterior, donde se parte de la suposición de que existe una sociedad sana y armónica a la cual el sujeto, una vez cumplida su pena, debe adaptarse. Al mismo tiempo, esa noción de integración no interpela al sujeto desde un trazo único de personalidad, sino que permite un despliegue subjetivo diverso, más amplio.

En razón de las dificultades e insuficiencias señaladas, este camino necesario de transformaciones se supone arduo y extenso, donde no solo se debe ejercer la crítica y proponer alternativas al encarcelamiento como herramienta penal única y privilegiada, sino también construir consenso social en torno a ese posible nuevo paradigma a construir. Esta última tarea en particular, se enfrenta también al desmanejo mediático del tema de la seguridad y al denominado populismo punitivo, ya mencionado (KESSLER, 2010), que alimentan la demanda social de mayores y más extensas penas para un número cada vez más 
amplio de tipos penales.

Hasta que esta posibilidad de cambios tome forma institucional y adquiera la hegemonía necesaria para su existencia, es necesario producir formas de intervención dirigidas a la población penitenciaria desde el campo de la salud mental comunitaria, que atiendan a sus numerosas demandas y necesidades. Las mismas no pueden escindirse de las condiciones sociales e institucionales de estos sujetos, lo que implica un límite significativo para este tipo de intervenciones. No obstante, y desde la posibilidad de atenuar y contener a algunos de los efectos del encierro, ciertas acciones que favorezcan la creación de formas de sociabilidad más tolerantes en esos contextos pueden ser formuladas, partiendo del principio de que la cárcel inhibe ciertas formas de expresión y vínculo posibles de ser promovidas desde una intervención que rescate aspectos vinculares afectada por la alienación institucional.

Para esto, no hay una técnica definida, sino que se trata de sostener un principio elemental de las políticas aquí apuntadas: la escucha abierta y la construcción de propuestas de intervención que atiendan las demandas y rescaten los deseos y condiciones de cada sujeto o grupo.

Se toma distancia aquí del remanido concepto de resiliencia, dado que éste se suele apoyar en la suposición de una condición íntima resiliente, como una virtud individual e intrínseca (TRUFFINO, 2010). En cambio, el término resistencia refiere también a una dimensión grupal e histórica y se apoya en la existencia de un poder opresor contra el cual dicha acción se produce, pudiendo hacerse de forma individual y/o colectiva. En definitiva, se trata de construir espacios de reflexión, de carácter protectivo, donde cada sujeto o grupo encuentre condiciones para resistir a la agresión institucional sufrida.

La educación en contextos carcelarios (ONOFRE \& JULIÃO 2013), puede ser una herramienta poderosa en ese sentido, en tanto no se reduzca a una mera transmisión de conocimientos escolares, a la manera de la educación bancaria que Freire (1993) criticó.

\section{Apuntes finales para una propuesta inconclusa}

Por lo expuesto, se considera que el modelo actual de resocialización hace parte de un tipo de ins- titución condenada al fracaso, que al mismo tiempo parece gozar de excelente salud y no para de autoreproducirse.

Como también fue señalado, desde la salud mental comunitaria se puede sostener una crítica radical a esos dispositivos, aún si no se dispone de una propuesta alternativa acabada para dichos modelos. La crítica en sí, desprovista de propuestas, tiene el potencial de generar a futuro la formulación de esas alternativas, siendo esta una exigencia ética sujeta a los tiempos inciertos propios de cualquier construcción política.

La situación actual, derivada de la pandemia y sus consecuencias sociales, como el encierro y los límites para la vida social plena, puede servir como una referencia interesante para pensar los efectos del encierro y del deterioro de los lazos sociales cotidianos, capaz quizás de interpelar la manera en que la cárcel reproduce dichos efectos a partir de suponer un beneficio posible en los mismos para quien los sufre.

Estas posibles transformaciones son parte inseparable de cambios más amplios, que transiten en el sentido de la promoción de una sociedad más justa y equitativa.

\section{Referencias bibliográficas}

AGUIRRE, Carlos. "Cárcel y sociedad en América Latina”. In: Historia social urbana: Espacios y flujos. p. 209-252, 2009.

BANCO INTERAMERICANO DE DESARROLLO (BID) (2020). Las cárceles de América Latina y El Caribe ante la crisis sanitaria del COVID-19. Disponible en: https://publications.iadb.org/publications/ spanish/document/Las-carceles-de-America-Latina-y-el-Caribe-ante-la-crisis-sanitaria-del-COVID-19. pd Accesado el 20 de enero del 2021.

BASAGLIA, Franco. La Institución Negada. Barcelona, Biblioteca Breve de Barral, 1972.

BEDRIÑANA, Fanny Tania, et. al. "Salud mental en prisión. Las paradojas socioeducativas". In: EduPsykhé: Revista de psicología y psicopedagogía, vol. 16, n 1, p. 98-116, 2017.

BRAVO, Omar \& CASTRILLÓN, María. "Vulnerabilidad en sujetos en situación de prisión domiciliaria 
en el Distrito Federal, Brasil”. In: Psicología desde el Caribe, vol. 25, p. 108-127, 2010.

BRAVO, O. "Saber, poder, salud. apuntes desde la cárcel”. In: Valeria Villegas, G.; Madriz, G. (Orgs). Filosofías del buen vivir, del mal vivir y otros ensayos. Caracas: Del Solar, 2012.

-----. "Estrategias comunitarias de prevención en salud en la cárcel de Villahermosa". In: Drugs and Addictive Behavior (histórico), vol.1, n², p. 156-170, 2016.

-----. "Instituciones carcelarias y efectos subjetivos". In: Bravo, O. Perspectivas multidisciplinarias sobre las cárceles: Una aproximación desde Colombia y América Latina. Editorial Universidad Icesi, 2018.

CALVO, Estibaliz. "El encierro carcelario. Impacto en las emociones y los cuerpos de las mujeres presas". In: Cuadernos de Trabajo Social, vol. 27, n², p. 395404, 2014.

CARRANZA, E. "Introducción". In: Bravo, O. Perspectivas multidisciplinarias sobre las cárceles: Una aproximación desde Colombia y América Latina. Editorial Universidad Icesi, 2018.

CESANO, José. "El tratamiento penitenciario. Derecho penal y procesal penal". In: Revista Nova tesis, vol. $1, \mathrm{n}^{\circ} 1$, p. 65-77, 2007.

DE LA CUESTA ARZAMENDI, José Luis. "La resocialización: objetivo de la intervención penitenciaria" In: Papers d'estudis i formació, vol. 12, p. 9, 1993.

DESVIAT, Manuel. Cohabitar la diferencia: de la reforma psiquiátrica a la salud mental colectiva. Grupo 5, Madrid, 2016.

FREIRE, Paulo. Pedagogía de la esperanza: un reencuentro con la pedagogía del oprimido. Buenos Aires: Siglo XXI, 1993.

GALENDE, Emiliano. Psicoanálisis y Salud Mental. Editorial Paidós, Buenos Aires, 1990.

-----. De un horizonte incierto: Psicoanálisis y Salud Mental en la sociedad actual. Buenos Aires: Paidós, 1998.

GOFFMAN, Erving. Internados. Buenos Aires: Amorrortu, 2001.
GRILLO, Elio Gómez. "Las prisiones en Latinoamérica”. In: Anuario de derecho penal y ciencias penales, vol. 33, n³, p. 689-696, 1980.

JIMÉNEZ, Norberto. "La resocialización como fin de la pena - una frustración en el sistema penitenciario y carcelario colombiano". In: Caderno CRH, vol. 30. N ${ }^{\circ}$ 81, p. 539-560, 2017.

KESSLER, Gabriel. Sociología del delito amateur. Buenos Aires: Paidós, 2010.

INSTITUTO LATINOAMERICANO DE LAS NACIONES UNIDAS PARA LA PREVENCIÓN DEL DELITO Y EL TRATAMIENTO DEL DELINCUENTE (ILANUD). El sistema penitenciario ante la encrucijada producto de la crisis provocada por el Covid-19. Disponible en: https://www.mpd.gov.ar/index.php/ noticias-feed/4962-documento-de-ilanud-sobre-el-sistema-penitenciario-en-el-marco-de-la-pandemia. Accesado el 14 de febrero del 2021.

INSTITUTO PENITENCIARIO DE COLOMBIA (INPEC) Intramural. Disponible en: https://www. inpec.gov.co/estadisticas-/tableros-estadisticos Accesado el 8 de marzo del 2021.

LOMBRAÑA, Andrea. Territorios psiquiátricos de la prisión: los servicios de salud mental en cárceles federales desde una perspectiva etnográfica. №3, 2016. Disponible en: www. revistadeprisiones. com.

LOPERA-ECHAVARRÍA, Juan. "El concepto de salud mental en algunos instrumentos de políticas públicas de la Organización Mundial de la Salud." In: Revista Facultad Nacional de Salud Pública, vol. 32, p.11-20, 2014.

NIÑO, Asiri; DÍAZ, Diana; \& RAMÍREZ, Luisa. "Trastorno mental en el contexto carcelario y penitenciario". In: Carta comunitaria. vol. 25, n¹43, p. 77-88, 2017.

ONOFRE, Elenice, \& JULIÃO, Elionaldo. "A Educação na Prisão como Política Pública: entre desafios e tarefas". In: Educação \& Realidade. Vol 38, n²1, p. 51-69, 2013.

ORGANIZACIÓN DE LAS NACIONES UNIDAS. Reglas Mínimas de las Naciones Unidas para el Tratamiento de los Reclusos, Reglas Nelson Mandela. Resolución 70/175 de 17 de diciembre de 2015. Nueva York, 2015. Disponible en https://www.unodc.org/ 
documents/justice-and-prison-reform/Nelson Mandela_Rules-S-ebook.pdfAccesado el 14 de diciembre del 2020.

TRUFFINO, Javier. "Resiliencia: una aproximación al concepto". Revista de Psiquiatría y Salud mental. Vol. 3, nº, p.145-151, 2010. 